\title{
Addendum: Noncoding RNAs prevent spreading of a repressive histone mark
}

\author{
Claudia Keller, Raghavendran Kulasegaran-Shylini, Yukiko Shimada, Hans-Rudolf Hotz \& Marc Bühler
}

Nat. Struct. Mol. Biol. 20, 994-1000 (2013); published online 21 July 2013; addendum published after print 25 September 2013

It was brought to our attention that the DNA sequence that was annotated as SPNCRNA.95 in PomBase (http://www.pombase.org/) is the same as a genomic element referred to as IRC1-R in Cam et al., Nat. Genet. 37, 809-819, 2005. Therefore, the BORDERLINE-encoding sequence and the IRC1-R element do partially overlap (Fig. 1). Correct annotations and coordinates have been updated in PomBase accordingly.

We note that, when Swi6 is overexpressed, the IRC1 DNA sequence is required to stop heterochromatin spreading on the left side of centromere 1 (IRC1-L; Noma et al., Cell 125, 859-872, 2006), but it remains to be investigated whether the same applies to the cen1-R boundary when IRC1-R is deleted and Swi6 overexpressed. Because heterochromatin spreading in our paper was assessed under normal Swi6 expression levels, we conclude that noncoding RNA (ncRNA)-mediated boundary activity is sufficient to stop the spread of heterochromatin under physiological conditions, although overlapping or complementary mechanisms may exist.

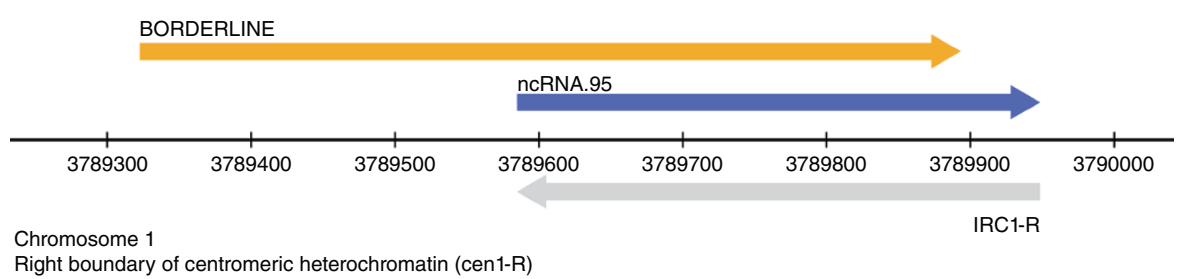

Figure 1 Schematics of BORDERLINE and ncRNA.95. Genomic locations of BORDERLINE (yellow) and ncRNA.95 (blue) on Schizosaccharomyces pombe chromosome 1, showing partial overlap of the elements. The sequence of the ncRNA.95 is the same as that of IRC1-R (gray). 